\title{
High power multi-color OPCPA source with simultaneous femtosecond deep-UV to mid-IR outputs
}

\author{
M. Baudisch, ${ }^{1, *}$ B. Wolter, ${ }^{1}$ M. Pullen, ${ }^{1}$ M. Hemmer, ${ }^{1,3}$ And J. Biegert ${ }^{1,2}$ \\ ${ }^{1}$ ICFO-Institut de Ciencies Fotoniques, The Barcelona Institute of Science and Technology, 08860 Castelldefels (Barcelona), Spain \\ ${ }^{2}$ ICREA-Institució Catalana de Recerca i Estudis Avançats, 08010 Barcelona, Spain \\ ${ }^{3}$ Center for Free-Electron Laser Science, Deutsches Elektronen Synchrotron (DESY), Hamburg, Germany \\ *Corresponding author: Matthias. Baudisch@icfo.eu
}

Received 3 June 2016; revised 3 July 2016; accepted 5 July 2016; posted 7 July 2016 (Doc. ID 267629); published 27 July 2016

\begin{abstract}
Many experimental investigations demand synchronized pulses at various wavelengths, ideally with very short pulse duration and high repetition rate. Here we describe a femtosecond multi-color optical parametric chirped pulse amplifier (OPCPA) with simultaneous outputs from the deep-UV to the mid-IR with optical synchronization. The high repetition rate of $160 \mathrm{kHz}$ is well suited to compensate for low interaction probability or low cross section in strong-field interactions. Our source features high peak powers in the tens to hundreds of MW regime with pulse durations below $110 \mathrm{fs}$, which is ideal for pump-probe experiments of nonlinear and strong-field physics. We demonstrate its utility by strong-field ionization experiments of xenon in the near- to mid-IR. () 2016 Optical Society of America
\end{abstract}

OCIS codes: (190.4970) Parametric oscillators and amplifiers; (320.7090) Ultrafast lasers.

http://dx.doi.org/10.1364/OL.41.003583

Improving our understanding of chemical dynamics, biological transformations, and charge carrier mobility is central to the advancement of science and technology in a wide range of areas. Ultrafast multi-color pump-probe techniques are important tools for such investigations, such as charge carrier dynamics in molecules [1] and semiconductors [2] or nondestructive material examination [3]. Typically, visible or near-IR pulses are used for carrier excitation, while mid-IR $(3-8 \mu \mathrm{m})$ outputs are of special interest as probes, due to the numerous vibrational and rotational molecular resonances in this spectral region. The employment of additional high-energy photons in the ultraviolet (UV) spectral range even enables the investigation of molecular transformations, such as photo-isomerization [4] and photo-dissociation [5].

Key parameters for all optical pump-probe experiments are the performance and capability of the light source. For instance, a high repetition rate is desirable for fast acquisition and various synchronized pulses at different wavelengths ranging from the UV to the mid-IR. Nonlinear pump-probe techniques, such as coherent anti-stokes Raman scattering (CARS) [6] or nonlinear fluorescence spectroscopy [7], require high pulse peak power. Hence, typical systems for such demanding applications are based on Ti:sapphire amplifiers that achieve the different output wavelengths by cascaded down-conversion [8,9] or white light generation [10-12], which requires spectral separation, compression of individual channels, and coherent recombination.

White light seeded sources, particularly electric field synthesizers, have demonstrated their potential for arbitrary waveform synthesis $[10,11]$, but their spectral coverage into the deep-UV or mid-IR range is limited. Furthermore, they struggle to provide high-energy, femtosecond pulses at repetition rates beyond the $\mathrm{kHz}$ regime. Operation at high repetition rates is, however, a requirement for a high signal-to-noise data acquisition and is especially important for studies on low cross section processes, such as electron recollision and recombination in strong-field physics [13], among others.

Here, we present a solution which encompasses all optical synchronization and multiple sub-110-fs outputs with wavelengths ranging from 270 to $3100 \mathrm{~nm}$ at a repetition rate of $160 \mathrm{kHz}$. Our source is a combination of a state-of-the-art, mid-IR optical parametric chirped pulse amplifier (OPCPA) [14-16] with a high-efficiency, cascaded up-conversion chain (setup shown in Fig. 1). The system permits the simultaneous generation of coherent output pulses at $3100 \mathrm{~nm}, 1620 \mathrm{~nm}$, $810 \mathrm{~nm}, 405 \mathrm{~nm}$, and $270 \mathrm{~nm}$ wavelengths with peak powers of $360 \mathrm{MW}, 156 \mathrm{MW}, 60 \mathrm{MW}, 31 \mathrm{MW}$, and $5 \mathrm{MW}$, respectively. All outputs from the UV to mid-IR can be focused to peak intensities of $10^{12}$ to $10^{14} \mathrm{~W} / \mathrm{cm}^{2}$. The root mean square (RMS) power stabilities of all outputs are measured to be below $0.7 \%$ over $30 \mathrm{~min}$, corresponding to 288 million shots. Compared to commonly used pump-probe sources [6-8], our OPCPA system shows a two order of magnitude higher repetition rate while permitting output peak intensities sufficient for driving strong-field physical processes.

The master oscillator is an erbium-based fiber laser with two outputs at 1030 and $1550 \mathrm{~nm}$ at a $100 \mathrm{MHz}$ repetition rate. The two outputs with sub-100 fs pulse duration are frequency mixed to a center wavelength of $3.1 \mu \mathrm{m}$ in a difference frequency generation (DFG) stage based on a magnesium oxide 


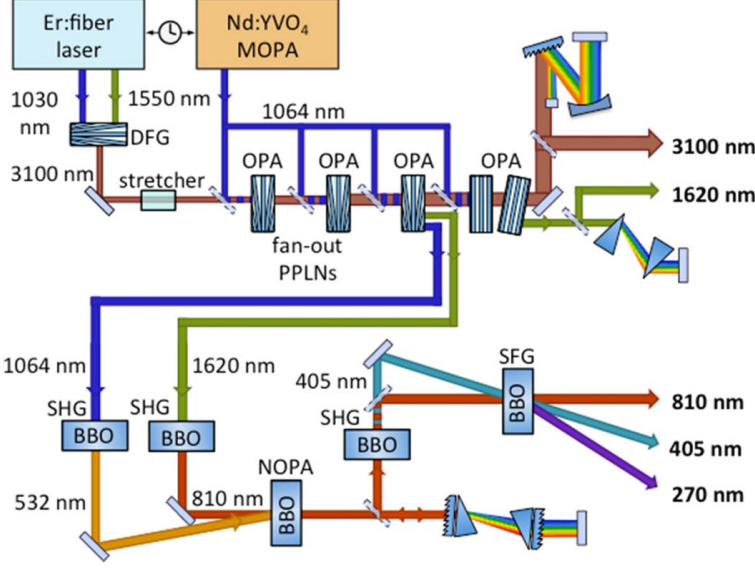

Fig. 1. Layout of high power optical parametric synthesizer. DFG: difference frequency generation; (N) OPA: (noncollinear) optical parametric amplification; SHG: second harmonic generation; SFG: sumfrequency generation.

doped, periodically poled lithium niobate (MgO:PPLN) crystal. The mid-IR output is passively carrier-envelope phase (CEP) stabilized $[17,18]$ and shows a $1 / e^{2}$ bandwidth of $900 \mathrm{~nm}$, supporting a $27 \mathrm{fs}$ transform limited pulse. The pump laser is a neodymium-doped yttrium vanadate (Nd:YVO4)-based master oscillator power amplifier (MOPA) system, which provides a pulse energy of $1 \mathrm{~mJ}$ with 9 ps pulse duration at $160 \mathrm{kHz}$ repetition rate. The pump pulses amplify the seeded mid-IR radiation in four consecutive $\mathrm{MgO}: \mathrm{PPLN}$ based (noncollinear) optical parametric amplification (OPA) stages to a maximum pulse energy of $40 \mu \mathrm{J}$. After amplification, the pulses are compressed to $55 \mathrm{fs}$ FWHM at a pulse energy of $20 \mu \mathrm{J}$ at $160 \mathrm{kHz}$. Parametric amplification can suffer from parametric generation background which would deteriorate the pulse quality. This occurs when amplification factors beyond $10^{5}$ are chosen for our conditions. When blocking the seed, the overall output of the OPCPA, for signal and idler, showed no measurable contribution above the noise floor of $35 \mathrm{~dB}$. To reduce pulse duration further, we have shown that the five cycle pulse duration can be reduced even further by means of clean and reproducible self compression directly in the mid-IR to $32 \mathrm{fs}$ (three optical cycles) in bulk dielectrics [19]. The performance of the system and its utility was demonstrated for a range of strong-field investigations [20] at focused peak intensities of up to $2 \times 10^{14} \mathrm{~W} / \mathrm{cm}^{2}$.

In the following, we describe the derivation of other short pulse outputs and the values of their performance parameters. The near-IR output of the OPCPA system is centered at $1620 \mathrm{~nm}$ and is obtained after the fourth mid-IR OPA stage as a byproduct of the three-wave-mixing parametric process. This beam is separated by means of a dichroic filter and subsequently compressed in a folded silica prism compressor down to $96 \mathrm{fs}$ pulse duration. After compression, the energy of the near-IR pulses is $15 \mu \mathrm{J}$, resulting in a peak power of $156 \mathrm{MW}$.

We pick $8 \mu \mathrm{J}$ of the third OPA's signal at $1620 \mathrm{~nm}$ and frequency double the chirped pulse in an antireflection (AR)-coated, $2 \mathrm{~mm}$-thick beta-barium borate (BBO) crystal cut at $19.8 \mathrm{deg}$. With a conversion efficiency of $42 \%, 3.4 \mu \mathrm{J}$ of near-IR energy, centered at $810 \mathrm{~nm}$, is generated. The $810 \mathrm{~nm}$ pulse is amplified further in a noncollinear parametric amplifier (NOPA) stage using an AR-coated $5 \mathrm{~mm}$-long BBO crystal cut at $23.5 \mathrm{deg}$. We use $190 \mu \mathrm{J}$ of residual pump at $1064 \mathrm{~nm}$ from the third OPA and double it to $114 \mu \mathrm{J}$ at $532 \mathrm{~nm}$ for pumping the NOPA. In the NOPA, the initial $3.4 \mu \mathrm{J}$ at $810 \mathrm{~nm}$ are amplified to $15.4 \mu \mathrm{J}$, corresponding to a total conversion efficiency of $16 \%$. The stretched 3 ps long pulses at $810 \mathrm{~nm}$ are then compressed with a prism compressor consisting of $1200 \mathrm{l} / \mathrm{mm}$ transmission gratings and AR-coated BK7 prisms (apex angle of $56 \mathrm{deg}$ ). We measure a compressed pulse duration of $51 \mathrm{fs}$ at $810 \mathrm{~nm}$ with pulse energy of $5.3 \mu \mathrm{J}$.

In order to reach shorter wavelengths in the UV, we rely on frequency up-conversion of the $810 \mathrm{~nm}$ output. An AR-coated $0.2 \mathrm{~mm}$-thick BBO crystal cut at $29.2 \mathrm{deg}$ is used for frequency doubling of the $810 \mathrm{~nm}$ pulse to reach $1.9 \mu \mathrm{J}$ energy at $405 \mathrm{~nm}$ (35\% conversion efficiency). We use self-diffraction frequencyresolved optical gating (FROG) to characterize the $400 \mathrm{~nm}$ pulses and retrieve a pulse duration of $47 \mathrm{fs}$. Reaching further into the UV is achieved by noncollinear sum-frequency generation (SFG) of the $405 \mathrm{~nm}$ and $810 \mathrm{~nm}$ pulses in a $0.2 \mathrm{~mm}$-thick $\mathrm{BBO}$ crystal cut at $44 \mathrm{deg}$. We measure $0.5 \mu \mathrm{J}$ of deep-UV light centered at $270 \mathrm{~nm}$. The pulse duration at $270 \mathrm{~nm}$ is measured with two-photon absorption autocorrelation [21] to $109 \mathrm{fs}$. After the last SFG stage, $3.1 \mu \mathrm{J}$ at $810 \mathrm{~nm}, 1.5 \mu \mathrm{J}$ a $405 \mathrm{~nm}$, and $0.5 \mu \mathrm{J}$ at $270 \mathrm{~nm}$ are available, resulting in peak powers of $60 \mathrm{MW}$, $31 \mathrm{MW}$, and $5 \mathrm{MW}$, respectively. Figure 2 shows in the inset
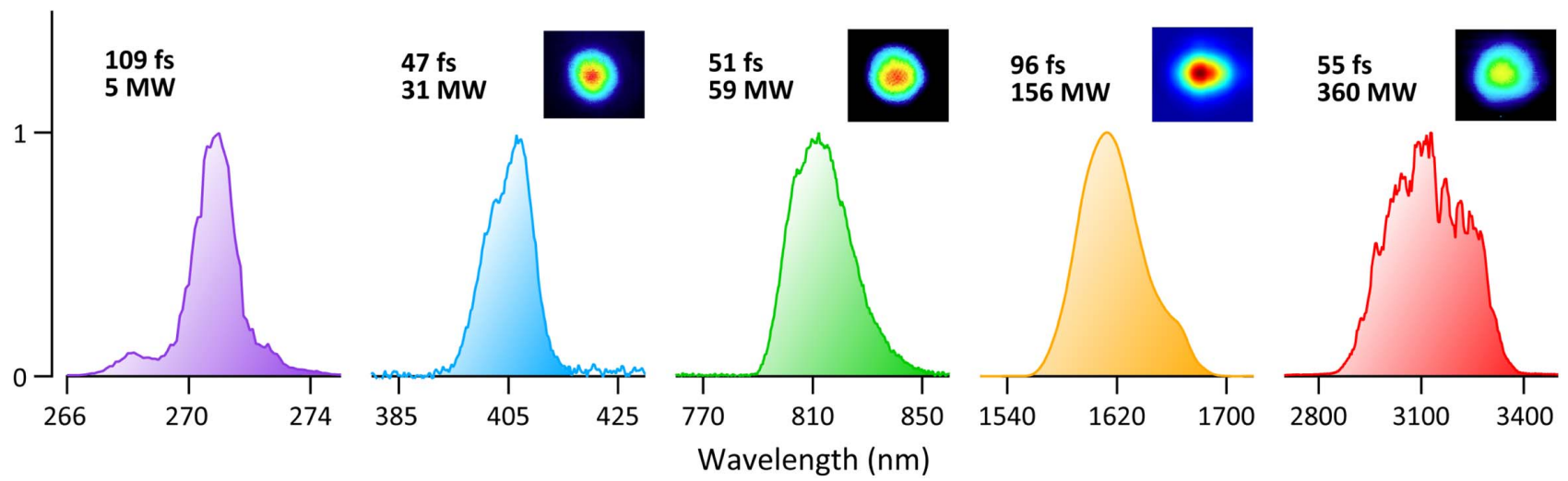

Fig. 2. Measured pulse energies and spectra of all outputs of the optical parametric synthesizer. In the insets the measured beam profiles are shown, respectively, obtained by a Si-CCD camera (for 400 and $800 \mathrm{~nm}$ ), by a scanning slit beam profiler (for $1600 \mathrm{~nm}$ ), and by a bolometer camera (for $3100 \mathrm{~nm}$ ). The beam profile of the output at $270 \mathrm{~nm}$ could not be measured due to the absence of a suitable camera. 


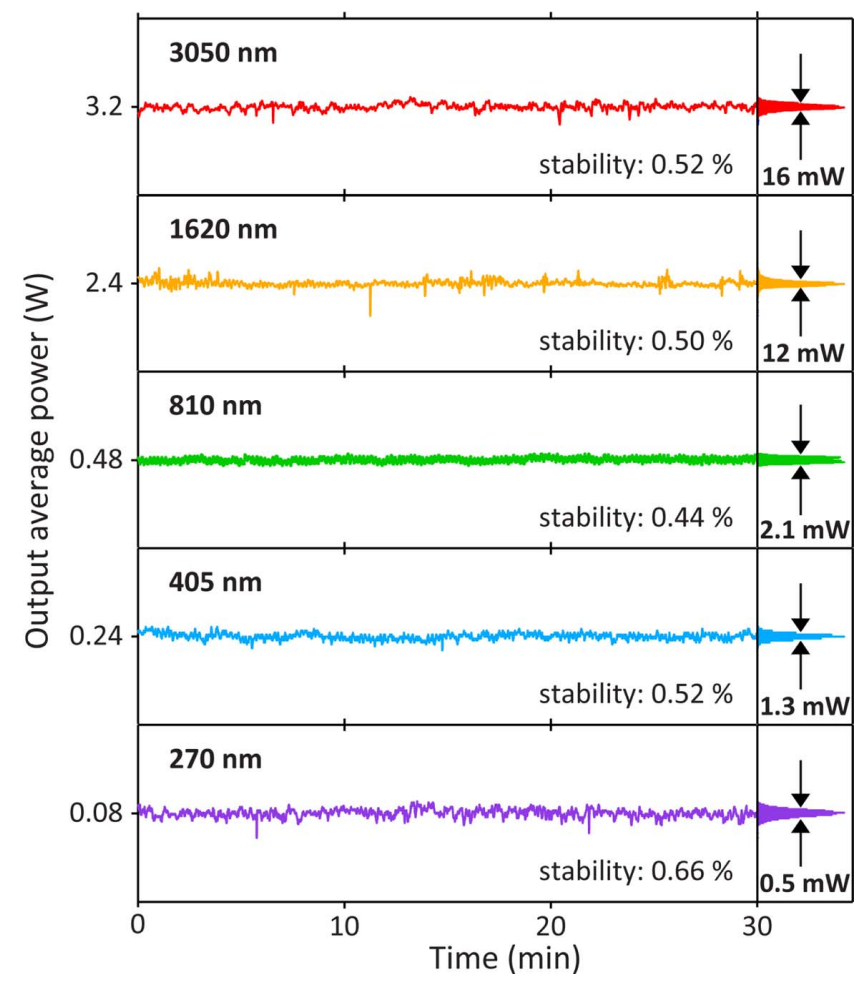

Fig. 3. Measured power stability of the OPCPA outputs over $30 \mathrm{~min}$. All multi-color outputs at 3050, 1620, 810, 405, and $270 \mathrm{~nm}$ center wavelength obtain power fluctuations below $0.7 \%$.

the beam profiles at the various wavelengths, which are all excellent, indicating good ability to focus to high peak intensity.

An equally important parameter of any system for experiments is the output stability, as this directly influences the signal-to-noise and the possible, or required, measurement time. The power stability is recorded with a pyroelectric detector to cover all wavelength ranges, and the RMS power fluctuations

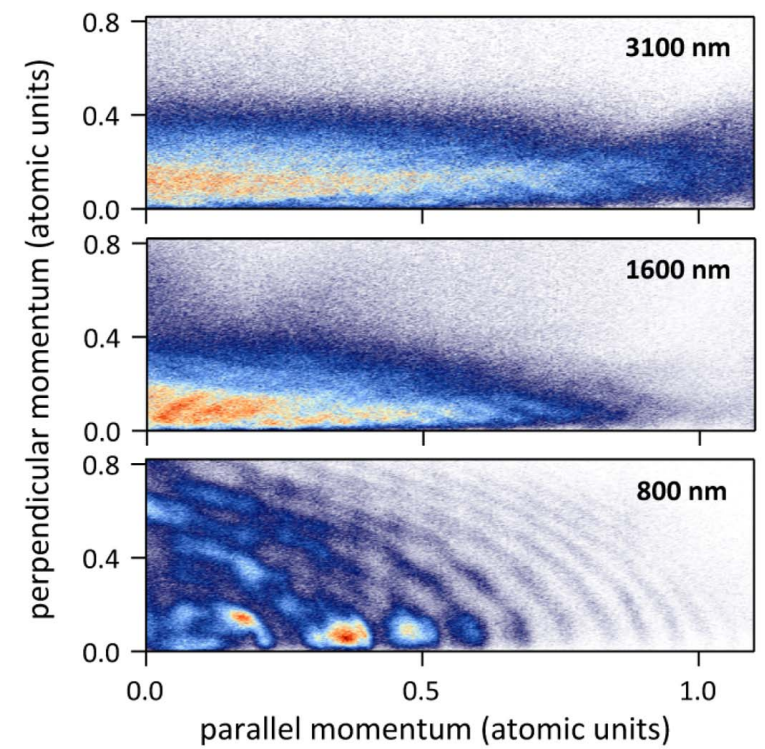

of all multi-color outputs are measured to below $0.7 \%$ over $30 \mathrm{~min}$ at $160 \mathrm{kHz}$ repetition rate (see Fig. 3). Comparing with typical $1 \mathrm{kHz}$ systems, we note that a measurement time of 30 min with our $160 \mathrm{kHz}$ system would be equivalent to 3.3 days measurement time.

Based on the excellent performance of the system, we turned to employing it for a strong-field investigation, which shows the range of different regimes, which are accessible for investigations from one single light source. We investigate the transition from multi-photon to tunneling ionization of xenon driven by the near- to mid-IR outputs with a reaction microscope (REMI) [22]. In our setup, the near- and mid-IR driving beams are focused by an on-axis, parabolic mirror with $50 \mathrm{~mm}$ focal length onto a supersonic xenon gas jet inside the REMI. We measure at $810 \mathrm{~nm}, 1600 \mathrm{~nm}$, and $3100 \mathrm{~nm}$ wavelengths to demonstrate how photoionization scales for these wavelengths and at peak intensities of $4.6 \times 10^{13}, 1.9 \times 10^{13}$, and $2.2 \times 10^{13} \mathrm{~W} / \mathrm{cm}^{2}$, respectively. The UV outputs were not used due to a lack of suitably coated refocusing optic inside the REMI.

In Fig. 4 (left), the extracted momentum maps correlated to single ionized xenon atoms are shown for the different driving wavelengths. The calculated kinetic energy distribution of the momentum maps $\left(E_{\text {kin }} \propto p_{\perp}^{2}+p_{\|}^{2}\right)$ can be seen in Fig. 4 (right). The momentum distribution of the liberated electrons driven at $810 \mathrm{~nm}$ contains a rich structure, while the momentum distributions for 1620 and $3100 \mathrm{~nm}$ are rather smooth. This difference is a direct result of the transition of the strong-field ionization regime that is characterized by the Keldysh parameter, $\gamma \propto 1 / \lambda$ [23]. For $\gamma>1$, multi-photon ionization is prominent, which results in the rich structure observed for $810 \mathrm{~nm}$, while for $\gamma \ll 1$, tunnel ionization dominates in the quasi-static regime, which results in the more continuous spectra observed for 1620 and $3100 \mathrm{~nm}$.

In the momentum plot for $810 \mathrm{~nm}(\gamma=1.47)$, the interference pattern for higher momentum can be attributed to intercycle interferences that result in structures known as above threshold ionization (ATI) rings [24,25], while the features at

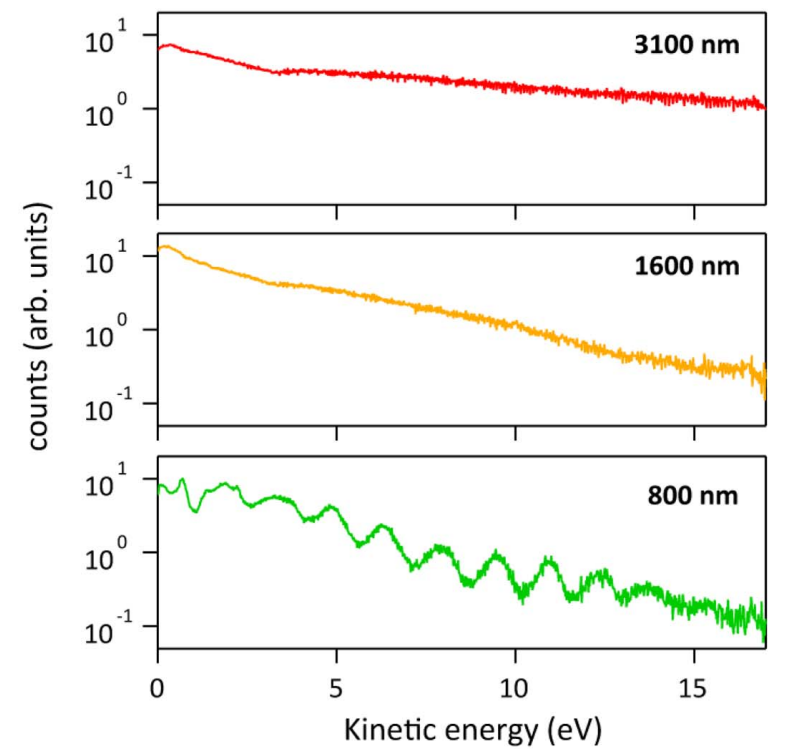

Fig. 4. (Left) Obtained momentum distribution of the liberated electrons corresponding to $\mathrm{Xe}^{+}$after strong-field ionization of xenon driven at 810,1620 , and $3100 \mathrm{~nm}$ center wavelengths. The data are plotted as a function of the parallel and perpendicular momentum with respect to the laser polarization. (Right) Corresponding electron kinetic energy distributions. 
smaller momenta are mainly attributed to intra-cycle quantum path interferences [26]. The ATI rings are a consequence of the multi-photon ionization effect, and every ring corresponds to ionization with a different number of photons, confirmed by the inter-ring spacing being equal to the photon energy of the driving laser. In the case of our $810 \mathrm{~nm}$ driving laser, the photon energy is $1.53 \mathrm{eV}$, which is in agreement with the peak spacing in the kinetic energy plot. In contrast, ionization with the $1620 \mathrm{~nm}$ $(\gamma=1.14)$ pulse occurs in a transition region toward the quasistatic regime. Finally, at $3100 \mathrm{~nm}(\gamma=0.55)$, ionization occurs in the quasi-static regime, and due to the nature of the tunneling process, where an electron is ejected with zero kinetic energy, we do not expect to resolve ATI rings - the overall distribution of the electron kinetic energies is therefore continuum-like.

The reported strong-field ionization measurements demonstrate the capability of our source to drive future high peak power pump-probe experiments with intensities up to $10^{14} \mathrm{~W} / \mathrm{cm}^{2}$. Although the transition of strong-field ionization from the multiphoton to tunneling regime has been studied previously $[27,28]$, in this Letter we show the first experimental investigation from the near-IR to the true mid-IR spectral region $(\lambda>3 \mu \mathrm{m})$ driven by a single light source at an unprecedented repetition rate of $160 \mathrm{kHz}$. The high repetition rate of the source is a key enabling factor to study low cross section processes, such as electron correlation in double or multiple ionization or recollision events, such as laser induced electron diffraction (LIED) $[29,30]$ and broadband laser-driven electron scattering [31]. These two examples are strong-field imaging techniques of the structure of the nucleus, which allow unprecedented spatial resolutions in the sub-Angstrom regime. The advantage of the described light source is the simultaneous synchronized output at various wavelengths, which now enables time-resolved investigation of electron and nuclear dynamics during photochemical reactions. A short wavelength pulse in the UV would be used to trigger a photochemical reaction, while the longer wavelength pulse would probe the reaction over time [32].

In conclusion, we demonstrate a new approach for a high peak power, all-coherent multi-color source supporting outputs from the deep-UV to the mid-IR with MW peak power levels. The high repetition rate of $160 \mathrm{kHz}$, the excellent power stability, and the optical synchronization makes the presented OPCPA system a key enabling tool for future UV-pump and IR-probe experiments, but also enables a variety of high peak power, time-resolved pump-probe experiments in the UV, visible, near-IR, and mid-IR spectral region. The fact that all outputs at 270, 405, 810,1620, and $3100 \mathrm{~nm}$ wavelength can be used simultaneously and obtain pulse durations below $110 \mathrm{fs}$, allows pump-probe temporal resolutions of below $150 \mathrm{fs}$, which is sufficient to resolve a large range of ultrafast dynamics. Future improvements to our system include modifications to obtain fully CEP-controlled outputs to enable true electric field waveform synthesis [10] or optimal control over electron recollision [33].

Funding. Ministerio de Economía y Competitividad (MINECO) (FIS2014-51478-ERC, FIS2014-56774-R); Severo Ochoa Programme for Centres of Excellence in R\&D (SEV-2015-0522); Catalan Agencia de Gestió d'Ajuts Universitaris i de Recerca (AGAUR) (SGR 2014-2016); Fundació Cellex Barcelona; European Union's Horizon 2020 research and innovation program Laserlab-Europe (654148);
Marie Sklodowska-Curie (641272); ICFONEST+ programme, partially funded by COFUND (FP7-PEOPLE-2013COFUND); Agència de Gestió d'Ajuts Universitaris i de Recerca (AGAUR) (FI-DGR 2013-2015).

\section{REFERENCES}

1. P. Hockett, C. Z. Bisgaard, O. J. Clarkin, and A. Stolow, Nat. Phys. 7, 612 (2011).

2. J. Cabanillas-Gonzalez, G. Grancini, and G. Lanzani, Adv. Mater. $\mathbf{2 3}$ 5468 (2011).

3. P. M. Norris, A. P. Caffrey, R. J. Stevens, J. M. Klopf, J. T. McLeskey, Jr., and A. N. Smith, Rev. Sci. Instrum. 74, 400 (2003).

4. Y. H. Jiang, A. Rudenko, O. Herrwerth, L. Foucar, M. Kurka, K. U. Kühnel, M. Lezius, M. F. Kling, J. van Tilborg, A. Belkacem, K. Ueda, S. Düsterer, R. Treusch, C. D. Schröter, R. Moshammer, and J. Ullrich, Phys. Rev. Lett. 105, 263002 (2010).

5. H. Sato, Chem. Rev. 101, 2687 (2001).

6. R. F. Begley, A. B. Harvey, and R. L. Byer, Appl. Phys. Lett. 25, 387 (1974).

7. A. Volkmer, D. A. Hatrick, and D. J. S. Birch, Meas. Sci. Technol. 8, 1339 (1997).

8. F. Seifert, V. Petrov, and M. Woerner, Opt. Lett. 19, 23 (1994).

9. V. Petrov, Prog. Quantum Electron. 42, 1 (2015).

10. A. Wirth, M. Th. Hassan, I. Grguraš, J. Gagnon, A. Moulet, T. T. Luu S. Pabst, R. Santra, Z. A. Alahmed, A. M. Azzeer, V. S. Yakovlev, V. Pervak, F. Krausz, and E. Goulielmakis, Science 334, 195 (2011).

11. S. W. Huang, G. Cirmi, J. Moses, K. H. Hong, S. Bhardwaj, J. R. Birge L. J. Chen, E. Li, B. J. Eggleton, G. Cerullo, and F. X. Kärtner, Nat. Photonics 5, 475 (2011).

12. C. Manzoni, D. Polli, and G. Cerullo, Rev. Sci. Instrum. 77, 023103 (2006)

13. D. R. Austin and J. Biegert, Phys. Rev. A 86, 023813 (2012).

14. O. Chalus, P. K. Bates, M. Smolarski, and J. Biegert, Opt. Express 17, 3587 (2009).

15. M. Hemmer, A. Thai, M. Baudisch, H. Ishizuki, T. Taira, and J. Biegert, Chin. Opt. Lett. 11, 013202 (2013).

16. M. Baudisch, H. Pires, H. Ishizuki, T. Taira, M. Hemmer, and J. Biegert, J. Opt. 17, 094002 (2015).

17. A. Baltuska, T. Fuji, and T. Kobayashi, Phys. Rev. Lett. 88, 133901 (2002)

18. T. Fuji, A. Apolonski, and F. Krausz, Opt. Lett. 29, 632 (2004).

19. M. Hemmer, M. Baudisch, A. Thai, A. Couairon, and J. Biegert, Opt. Express 21, 28095 (2013).

20. B. Wolter, M. G. Pullen, M. Baudisch, M. Sclafani, M. Hemmer, A. Senftleben, C. D. Schröter, J. Ullrich, R. Moshammer, and J. Biegert, Phys. Rev. X 5, 021034 (2015).

21. C. Homann, N. Krebs, and E. Riedle, Appl. Phys. B 104, 783 (2011)

22. R. Moshammer, M. Unverzagt, W. Schmitt, J. Ullrich, and H. SchmidtBöcking, Nucl. Instrum. Methods Phys. Res. Sect. B 108, 425 (1996).

23. L. V. Keldysh, Zh. Eksp. Teor. Fiz. 47, 1945 (1964) [Sov. Phys. J. Exp. Theor. Phys. 20, 1307 (1965)].

24. P. Agostini, F. Fabre, G. Mainfray, G. Petite, and N. K. Rahman, Phys. Rev. Lett. 42, 1127 (1979).

25. F. Fabre, G. Petite, P. Agostini, and M. Clement, J. Phys. B 15, 1353 (1982).

26. D. G. Arbó, K. L. Ishikawa, E. Persson, and J. Burgdörfer, Nucl. Instrum. Methods Phys. Res. Sect. B 279, 24 (2012).

27. E. Mevel, P. Breger, R. Trainham, G. Petite, P. Agostini, A. Migus, J. P. Chambaret, and A. Antonetti, Phys. Rev. Lett. 70, 406 (1993).

28. L. Fechner, "Combined faculties of the natural sciences and mathematics," Ph.D. dissertation (Ruperto-Carola-University of Heidelberg, 2014), pp. 109.

29. C. I. Blaga, J. Xu, A. D. DiChiara, E. Sistrunk, K. Zhang, P. Agostini, T. A. Miller, L. F. DiMauro, and C. D. Lin, Nature 483, 194 (2012).

30. M. G. Pullen, B. Wolter, A. T. Le, M. Baudisch, M. Hemmer, A. Senftleben, C. D. Schröter, J. Ullrich, R. Moshammer, C. D. Lin, and J. Biegert, Nat. Commun. 6, 7262 (2015).

31. J. Xu, C. I. Blaga, K. Zhang, Y. H. Lai, C. D. Lin, T. A. Miller, P. Agostini, and L. F. DiMauro, Nat. Commun. 5, 4635 (2014).

32. H. Stapelfeldt and T. Seideman, Rev. Mod. Phys. 75, 543 (2003).

33. L. E. Chipperfield, J. S. Robinson, J. W. G. Tisch, and J. P. Marangos, Phys. Rev. Lett. 102, 063003 (2009). 